\title{
Outcome After Steroid Withdrawal in Adult Renal Transplant Patients Receiving Tacrolimus-Based Immunosuppression
}

\author{
P. Chakrabarti, H.Y. Wong, A. Toyofuku, V.P. Scantlebury, M.L. Jordan, C. Vivas, A.B. Jain, \\ J. McCauley, J. Johnston, P.S. Randhawa, T.R. Hakala, R.L. Simmons, J.J. Fung, T.E. \\ Starzl, and R. Shapiro \\ Thomas E. Starzl Transplantation Institute, Division of Urologic Surgery, Division of Nephrology, \\ Department of Pathology, and Division of General and Vascular Surgery, University of Pittsburgh, \\ Pittsburgh, PA 15213, USA
}

Corticosteroids, in spite of their well-known side effects, have essentially always been an integral part of immunosuppressive regimens in renal transplantation. With the introduction of tacrolimus, however, steroid withdrawal has been possible in some $70 \%$ of successfully transplanted adults and children. ${ }^{1-8}$ An important question, however, concerns the outcome after steroid withdrawal in adult patients on tacrolimus. We looked at our adult renal transplant patients receiving tacrolimus-based immunosuppression and examined the short- and mediumterm outcomes after steroid withdrawal.

\section{PATIENT AND METHODS}

Between August 1991 and December 1996, 795 renal transplants were performed in adult patients receiving tacrolimus-based immunosuppression at the University of Pittsburgh. 43 $(5 \%)$ of these grafts were lost within 3 weeks of transplantation, and the recipients were still on steroids at the time of graft loss, and 4 were lost to follow-up; both groups were excluded from further analysis. The mean recipient age was $46 \pm 14$ years (range 18-84). Five hundred fifty-four $(74 \%)$ were primary transplants, and $194(26 \%)$ were second $(n=144)$, third $(n=$ $39)$, or fourth $(n=11)$ transplants. Four hundred forty-two (59\%) were males and $306(41 \%)$ were females. Six hundred three (81\%) were whites, $116(15 \%)$ were African-Americans, and 29 (4\%) were Asians. The mean PRA was $11 \pm 22.5 \%$ (range 0 to 100 ).

The mean donor age was $36 \pm 19$ years (range 0.1 to 77 ). Six hundred fifty-six (88\%) kidneys were from cadaveric donors. The mean cold ischemia time was $30.7 \pm 9.5$ hours. There were $92(12 \%)$ living donors. The mean number of HLA matches and mismatches was $2.7 \pm 1.4$ and $2.9 \pm 1.5$, respectively. The mean number of DR-matches and mismatches was $1.1 \pm 0.6$ and $0.8 \pm 0.7$, respectively.

Immunosuppression was with tacrolimus and steroids with or without azathioprine, mycophenolate mofetil, or a one week course of low-dose cyclophosphsmide, as previously described. ${ }^{1-3}$ Induction antilymphocyte antibodies were generally not used. Although there were no formal a priori inclusion or exclusion criteria for complete withdrawal, steroids were tapered and discontinued in patients who maintained stable allograft function as the steroid dosage was gradually decreased. Steroids were restarted in recipients who experienced a rising serum creatinine with biopsy-proven acute rejection. Allograft failure was defined by return to dialysis, retransplantation, or death. 
Patient survival was calculated from the date of kidney transplantation until death, and graft survival was calculated from the date of kidney transplantation until graft failure or patient death. Survival curves were generated using the Kaplan-Meier (Product limit) method and were compared using the log-rank test. Values are presented as mean $\pm \mathrm{SD}$. A time dependent Cox regression analysis for hazard assumption was done to compare graft survival in recipients who came off steroids versus those who never came off. A Cox time dependent covariate $\mathrm{z}(\mathrm{t})$ was modeled, so that $\mathrm{z}(\mathrm{t})=1$ if the patient was weaned off at least once, and $\mathrm{z}(\mathrm{t})=0$ if the patient was not weaned off by time $t$.

A multivariate multiple logistic regression analysis was performed to compare the effect of recipient sex, recipient race, delayed graft function, recipient PRA, total HLA-matches, totalHLA mismatches, donor age, and donor type (cadaveric vs living related) on steroid withdrawal. The means between groups were compared by one way analysis of variance (ANOVA). Catagorical data were analyzed by Pearson chi-square method. $\mathrm{P}$ values less than 0.05 were considered statistically significant.

\section{RESULTS}

Five hundred thirty-one (71\%) patients were taken off prednisone a median of 9.7 months after transplantation. Of these 531 patients, 398 (75\%) remained off prednisone (OFF), and 133 (25\%) were restarted on prednisone a median of 14.5 months after discontinuing steroids (OFF/ ON). Two hundred seventeen ( $29 \%$ ) were never taken off prednisone (ON). The mean followup was $4.6 \pm 2.6$ years. The 1,3 , and 5 year actuarial patient survival rates in the OFF group were $99.7 \%, 98 \%$, and $96 \%$, respectively; in the OFF/ON group, they were $99 \%, 98 \%$, and 93\%. ( $P=$ NS compared to the OFF group), and in the ON group, they were $91 \%, 82 \%$, and $78 \%$ ( $P=.0001$ compared to the OFF and OFF/ON groups). The 1,3 , and 5 year actuarial graft survival rates in the OFF group were $99.5 \%, 96 \%$, and $89 \%$ respectively; in the OFF/ON group, they were $98.5 \%, 89 \%$, and $71 \%$, and in the ON group, they were $74 \%, 52 \%$, and $42 \%(P=$. 0001). A Cox regression analysis showed that the hazard assumption for graft failure for those who came off prednisone was 0.124 relative to those who were never off prednisone $(P<$. 0001 ). Acute rejection-free survival was $65 \%$ in the OFF group compared to $20 \%$, and $24 \%$ in the $\mathrm{OFF} / \mathrm{ON}$ and $\mathrm{ON}$ groups respectively $(P<.05)$. The incidences of steroid resistant rejection episodes were $4 \%$ in the OFF group, and $12 \%$, and $22 \%$ in the OFF/ON, and ON groups respectively $(P<.001)$. There were $82(21 \%)$ retransplants in the OFF group, $33(25 \%)$ in the OFF/ON group, and $66(30 \%)$ in the ON group $(P<.001)$. The mean PRA was higher in the ON group $14.9 \pm 26 \%$, than in the OFF group, $9.4 \pm 20.6 \%$, and the OFF/ON group, $10.2 \pm 20.4 \%(P=.01)$. There was no effect of donor race and sex, recipient race and sex, cadaveric vs living donor, 48-hour graft function, and total HLA mismatches or matches on the likelihood of being weaned off steroids. In the OFF group, the serum creatinine at most recent follow-up was $1.8 \pm 1.7 \mathrm{mg} / \mathrm{dL}$; in the $\mathrm{OFF} / \mathrm{ON}$ and $\mathrm{ON}$ groups, it was $3.7 \pm 2.9 \mathrm{mg} / \mathrm{dL}$ and $4.5 \pm 3.8 \mathrm{mg} / \mathrm{dL}$, respectively $(P=.0001)$. In the OFF group, the hematocrit at most recent follow-up was $40 \pm 7.2$; whereas, in the $\mathrm{OFF} / \mathrm{ON}$ and $\mathrm{ON}$ groups, it was $35 \pm 7.3$, and $33 \pm 7.9$ respectively $(P=.01)$. The mean tacrolimus level in the OFF group was $8.2 \pm 3.9 \mathrm{ng} / \mathrm{mL}$ at last follow-up; in the OFF/ON and ON groups it was $8.3 \pm 4.1 \mathrm{ng} / \mathrm{mL}$, and $10.5 \pm 6.6 \mathrm{ng} / \mathrm{mL}$, respectively $(P=.03)$.

\section{DISCUSSION}

The primary goal of this analysis was to assess the safety of steroid withdrawal in our adult renal transplant recipients receiving tacrolimus-based immunosuppression. The main questions for us concerned the safety of steroid withdrawal and, more importantly, the outcomes in patients who were withdrawn from and then resumed steroids. It should be emphasized that patients were not randomly assigned to steroid withdrawal, and patients who 
never came off $(\mathrm{ON})$, and those who were withdrawn from and then resumed steroids (OFF/ $\mathrm{ON}$ ), had a number of risk factors, including retransplantation, sensitization, and acute rejection. Five year patient survival rates were essentially the same in the OFF and the OFF/ ON groups, but 5-year graft survival rates were significantly lower in the OFF/ON group compared to the OFF group. The incidence of rejection and the mean serum creatinine were higher in the OFF/ON group, and these findings are of concern with regard to long term followup.

To summarize, these data suggest that steroid withdrawal in adult renal transplant patients receiving tacrolimus-based immunosuppression is associated with reasonable medium-term patient and graft survival and reasonable graft function. The patients who discontinued and then resumed steroids had comparable patient survival, but lower graft survival and worse renal function. The patients who never discontinued prednisone had the worst outcome.

\section{References}

1. Shapiro R, Jordan ML, Scantlebury VP, et al. Transplantation Proceedings 1996;28:2117. [PubMed: 8769173]

2. Shapiro R. Transplantation Proceedings 1997;29:45. [PubMed: 9123077]

3. Shapiro R, Jordan ML, Scantlebury VP, et al. Transplantation Proceedings 1998;30:1375. [PubMed: 9636557]

4. Chakrabarti P, Wong HY, Scantlebury VP, et al. Transplantation. (in press).

5. Shapiro R, Scantlebury VP, Jordan ML, et al. Transplantation 1999;67:299. [PubMed: 10075598]

6. Shapiro R, Scantlebury VP, Jordan ML, et al. Transplantation 1996;62:1752. [PubMed: 8990356]

7. Grewal HP, Thistlethwaite JR Jr, Loss GE, et al. Transplantation Proceedings 1998;30:1378. [PubMed: 9636558]

8. Woodle ES. Transplantation Proceedings 1999;31:247. [PubMed: 10083094]

Transplant Proc. Author manuscript; available in PMC 2010 November 4. 